\title{
A PRIMALITY CRITERION BASED ON A LUCAS' CONGRUENCE
}

\author{
ROMEO MEŠTROVIĆ
}

ABSTRACT. Let $p$ be a prime. In 1878 É. Lucas proved that the congruence

$$
\left(\begin{array}{c}
p-1 \\
k
\end{array}\right) \equiv(-1)^{k} \quad(\bmod p)
$$

holds for any nonnegative integer $k \in\{0,1, \ldots, p-1\}$. The converse statement was given in Problem 1494 of Mathematics Magazine proposed in 1997 by E. Deutsch and I.M. Gessel. In this note we generalize this converse assertion by the following result: If $n>1$ and $q>1$ are integers such that

$$
\left(\begin{array}{c}
n-1 \\
k
\end{array}\right) \equiv(-1)^{k} \quad(\bmod q)
$$

for every integer $k \in\{0,1, \ldots, n-1\}$, then $q$ is a prime and $n$ is a power of $q$.

\section{INTRODUCTION AND THE MAIN RESULT}

As noticed in [6], many great mathematicians of the nineteenth century considered problems involving binomial coefficients modulo prime or prime power (for instance Babbage, Cauchy, Cayley, Gauss, Hensel, Hermite, Kummer, Legendre, Lucas, and Stickelberger). They discovered a variety of elegant and surprising theorems which are often easy to prove. For more information on these classical results, their extensions, and new results about this subject, see Dickson [3], and Granville [6]. Furthermore, the arithmetic and divisibility properties of binomial coefficients can be used to establish criteria for primality. In 1801 Gauss [4, Disquisitiones Arithmeticae, 1801, art. 329] wrote:

"The problem of distinguishing prime numbers from composite numbers ... is known to be one of the most important and useful in arithmetic. ... The dignity of the science itself seems to require that every possible means be explored for solution of a problem so elegant and so celebrated."

As far back as 1819 (see [6]), creator of of machines that were precursors of the modern computer Charles Babbage gave an easily proved characterization of the primes as follows: an integer $n \geq 2$ is a prime if and only if $\left(\begin{array}{c}n+m \\ n\end{array}\right) \equiv 1(\bmod n)$ for all positive integers $m$ with $0 \leq m \leq n-1$. This criterion was recently generalized by the author of this note in [10, Theorem 1.1]. In 1954 the amateur mathematician Pedro A. Piza [12] proved that an odd positive integer $2 n+1 \geq 3$

2010 Mathematics Subject Classification. 11A51, 11A07, 05A10, 11B65.

Keywords and phrases: primality criterion, congruence modulo a prime (prime power), Lucas' congruence, Lucas' theorem. 
is a prime if and only if $\left(\begin{array}{c}2 n-k \\ k-1\end{array}\right) \equiv 0(\bmod k)$ for all $k \in\{1,2, \ldots, n\}$. In 1972 H.B. Mann and D. Shanks [8] discovered another attractive primality criterion which may be stated as follows: a positive integer $k \geq 2$ is prime if and only if $\left(\begin{array}{c}n \\ k-2 n\end{array}\right) \equiv 0(\bmod k)$ for each $n \geq 1$ such that $k / 3 \leq n \leq k / 2$. The following "dual" criterion to that of Mann and Shanks was discovered in 1985 by H.W. Gould and W.E. Greig [5] in the following form: a positive integer $k \geq 2$ is a prime if and only if $\left(\begin{array}{c}-n \\ k-2 n\end{array}\right) \equiv 0(\bmod k)$ for each $n \geq 1$ such that $n \leq k / 2$. Notice that by the famous Lucas' theorem [7] given by the congruence (4), we immediately have $\left(\begin{array}{c}n p \\ m p\end{array}\right) \equiv\left(\begin{array}{c}n \\ m\end{array}\right)(\bmod p)$, where $p$ is a prime, $n$ and $m$ are integers with $0 \leq m \leq n$. In 2009 the author of this note [9. Theorem] proved a partial converse theorem of this assertion as follows: If $d, q>1$ are integers such that $\left(\begin{array}{c}n d \\ m d\end{array}\right) \equiv\left(\begin{array}{c}n \\ m\end{array}\right)(\bmod q)$ for every pair of integers $n \geq m \geq 0$, then $d$ and $q$ are powers of the same prime $p$.

Let $p$ be a prime. In 1878 É. Lucas [7] proved that

$$
\left(\begin{array}{c}
p-1 \\
k
\end{array}\right) \equiv(-1)^{k} \quad(\bmod p)
$$

for any nonnegative integer $k \in\{0,1, \ldots, p-1\}$. By Problem 1494 of Mathematics Magazine proposed by E. Deutsch and I.M. Gessel in 1997 [2] (see also [1, pp. 277-278]), a converse assertion is also true; that is, an integer $p \geq 2$ is a prime if and only if the congruence (1) holds for each $k \in\{0,1, \ldots, p-1\}$. T.-X. Cai and A. Granville [1, p. 277] proved that in this assertion the range for $k$ may be shortened to $0 \leq k \leq \sqrt{n}$. Accordingly, the simultaneous congruences (1) with $k \in\{0,1, \ldots, p-1\}$ could be used to identify primes.

Our Theorem 1 generalizes the previously mentioned criterion for primality. This is motivated by the following result.

Proposition 1. Let $p$ be a prime and let $f$ be a positive integer. Then for each $k \in\left\{0,1, \ldots, p^{f}-1\right\}$ we have

$$
\left(\begin{array}{c}
p^{f}-1 \\
k
\end{array}\right) \equiv(-1)^{k} \quad(\bmod p) .
$$

We are now ready to state the main result.

Theorem 1. Let $n>1$ and $q>1$ be integers such that

$$
\left(\begin{array}{c}
n-1 \\
k
\end{array}\right) \equiv(-1)^{k} \quad(\bmod q)
$$

for every integer $k \in\{0,1, \ldots, n-1\}$. Then $q$ is a prime and $n$ is a power of this prime $q$. 


\section{Proofs of Proposition 1 And Theorem 1}

Proof of Proposition 1] If $a=a_{0}+a_{1} p+\cdots+a_{l} p^{l}$ and $b=b_{0}+b_{1} p+\cdots+b_{l} p^{l}$ are the $p$-adic expansions of nonnegative integers $a$ and $b$ (so that $0 \leq a_{i}, b_{i} \leq p-1$ for all $i=0,1, \ldots, l)$, then by Lucas's theorem ([7]; also see [6] or [11]),

$$
\left(\begin{array}{l}
a \\
b
\end{array}\right) \equiv \prod_{i=0}^{l}\left(\begin{array}{l}
a_{i} \\
b_{i}
\end{array}\right) \quad(\bmod p)
$$

If we take $k=\sum_{i=0}^{f-1} k_{i} p^{i}$ with $0 \leq k_{i} \leq p-1$ for all $i=0,1, \ldots, f-1$, then in view of the fact $p^{f}-1=\sum_{i=0}^{f-1}(p-1) p^{i}$, the congruences (4) and (1) immediately yield

$$
\begin{aligned}
\left(\begin{array}{c}
p^{f}-1 \\
k
\end{array}\right) & =\left(\begin{array}{c}
\sum_{i=0}^{f-1}(p-1) p^{i} \\
\sum_{i=0}^{f-1} k_{i} p^{i}
\end{array}\right) \equiv \prod_{i=0}^{f-1}\left(\begin{array}{c}
p-1 \\
k_{i}
\end{array}\right) \quad(\bmod p) \\
& \equiv \prod_{i=0}^{f-1}(-1)^{k_{i}}=(-1)^{\sum_{i=0}^{f-1} k_{i}} \equiv(-1)^{k} \quad(\bmod p)
\end{aligned}
$$

Notice that in the last congruence of (5) we have used the fact that if $p$ is an odd prime, then $k$ and the sum $\sum_{i=0}^{f-1} k_{i}$ have the same parity, while for $p=2$ holds $1 \equiv-1(\bmod 2)$.

Proof of Theorem 1 is based on Proposition 1 and the following lemma.

Lemma 1. Let $p$ be a prime and let $f$ be a positive integer greater than 1 . Then

$$
\left(\begin{array}{c}
p^{f}-1 \\
p^{f-1}
\end{array}\right) \equiv \begin{cases}p-1 & \left(\bmod p^{2}\right) \text { if } p \geq 3 \\
3 & (\bmod 4) \text { if } p=2 .\end{cases}
$$

Proof. By using the identities $\left(\begin{array}{c}a-1 \\ b\end{array}\right)=\frac{a-b}{a}\left(\begin{array}{l}a \\ b\end{array}\right)$ and $\left(\begin{array}{l}a \\ b\end{array}\right)=\frac{a}{b}\left(\begin{array}{l}a-1 \\ b-1\end{array}\right)$ with $1 \leq b \leq a$, we have

$$
\begin{aligned}
\left(\begin{array}{c}
p^{f}-1 \\
p^{f-1}
\end{array}\right) & =\frac{p^{f}-p^{f-1}}{p^{f}}\left(\begin{array}{c}
p^{f} \\
p^{f-1}
\end{array}\right)=\frac{p^{f}-p^{f-1}}{p^{f}} \cdot \frac{p^{f}}{p^{f-1}}\left(\begin{array}{c}
p^{f}-1 \\
p^{f-1}-1
\end{array}\right) \\
& =(p-1)\left(\begin{array}{c}
p^{f}-1 \\
p^{f-1}-1
\end{array}\right)
\end{aligned}
$$

Further, we have

$$
\left(\begin{array}{c}
p^{f}-1 \\
p^{f-1}-1
\end{array}\right)=\prod_{i=1}^{p^{f-1}-1} \frac{p^{f}-i}{p^{f-1}-i}=\prod_{\substack{1 \leq i \leq p f-1-1 \\
i \neq 0(\bmod p)}} \frac{p^{f}-i}{p^{f-1}-i} \prod_{\substack{1 \leq i \leq p \\
i \equiv 0(\bmod p)}} \frac{p^{f}-i}{p^{f-1}-i}
$$

If $f \geq 3$, then

(9)

$$
\frac{p^{f}-i}{p^{f-1}-i} \equiv 1\left(\bmod p^{2}\right) \quad \text { for each } i \text { such that } 1 \leq i \leq p^{f-1}-1 \text { and } i \not \equiv 0(\bmod p) \text {. }
$$


Furthermore, for $f \geq 3$ we have

(10)

$$
\prod_{\substack{1 \leq i \leq p \\
i=0(\bmod p)}} \frac{p^{f}-i}{p^{f-1}-i}=\prod_{j=1}^{p^{f-2}-1} \frac{p^{f}-j p}{p^{f-1}-j p}=\prod_{j=1}^{p^{f-2}-1} \frac{p^{f-1}-j}{p^{f-2}-j}=\left(\begin{array}{c}
p^{f-1}-1 \\
p^{f-2}-1
\end{array}\right) .
$$

Substituting (9) and (10) into (8) we find that for each prime $p$ and every integer $f \geq 3$ holds

$$
\left(\begin{array}{c}
p^{f}-1 \\
p^{f-1}-1
\end{array}\right) \equiv\left(\begin{array}{c}
p^{f-1}-1 \\
p^{f-2}-1
\end{array}\right) \quad\left(\bmod p^{2}\right)
$$

Iterating the congruence (11) $f-2$ times yields

$$
\left(\begin{array}{c}
p^{f}-1 \\
p^{f-1}-1
\end{array}\right) \equiv\left(\begin{array}{c}
p^{2}-1 \\
p-1
\end{array}\right) \quad\left(\bmod p^{2}\right)
$$

which substituting into (7) for every $f \geq 3$ gives

$$
\left(\begin{array}{c}
p^{f}-1 \\
p^{f-1}
\end{array}\right) \equiv(p-1)\left(\begin{array}{c}
p^{2}-1 \\
p-1
\end{array}\right) \quad\left(\bmod p^{2}\right) .
$$

Further, for each prime $p \geq 3$ we have

$$
\begin{aligned}
\left(\begin{array}{c}
p^{2}-1 \\
p-1
\end{array}\right) & =\prod_{i=1}^{p-1} \frac{p^{2}-i}{p-i} \equiv \prod_{i=1}^{p-1} \frac{-i}{p-i} \quad\left(\bmod p^{2}\right) \\
& \equiv \prod_{i=1}^{p-1} \frac{-i(p+i)}{-i^{2}}=\prod_{i=1}^{p-1}\left(\frac{p}{i}+1\right) \equiv 1+p \sum_{i=1}^{p-1} \frac{1}{i} \quad\left(\bmod p^{2}\right) \\
& =1+p \sum_{i=1}^{(p-1) / 2}\left(\frac{1}{i}+\frac{1}{p-i}\right)=1+p^{2} \sum_{i=1}^{(p-1) / 2} \frac{1}{i(p-i)} \equiv 1 \quad\left(\bmod p^{2}\right) .
\end{aligned}
$$

Substituting (14) into (13) we obtain that for each $f \geq 3$ and any prime $p \geq 3$

$$
\left(\begin{array}{c}
p^{f}-1 \\
p^{f-1}
\end{array}\right) \equiv p-1 \quad\left(\bmod p^{2}\right)
$$

Notice also that for a prime $p \geq 3$ the identity (7) with $f=2$ and the congruence (14) yield

$$
\left(\begin{array}{c}
p^{2}-1 \\
p
\end{array}\right)=(p-1)\left(\begin{array}{c}
p^{2}-1 \\
p-1
\end{array}\right) \equiv p-1 \quad\left(\bmod p^{2}\right)
$$

The congruences (15) and (16) imply the first part of the congruence (6). 
It remains to consider the case when $p=2$. By (12), for each $f \geq 3$ we have

$$
\left(\begin{array}{c}
2^{f}-1 \\
2^{f-1}
\end{array}\right) \equiv 3 \quad(\bmod 4)
$$

which is also satisfied for $f=2$. The congruence (17) is in fact the second part of the congruence (6), and the proof is completed.

Proof of Theorem [1] Taking $k=1$ into the congruence (3) we obtain

$$
n \equiv 0(\bmod q) \text {. }
$$

Therefore, if $p$ is a prime divisor of $q$, then $n$ can be expressed as $n=s p^{f}$, where $f$ and $s$ are positive integers such that $s$ is not divisible by $p$. Now we consider the following three cases.

Case 1: $s=f=1$. Then $n=p$, and this together with the congruence (18) yields $q=p$.

Case 2: $s=1$ and $f \geq 2$. Then $n=p^{f}$, and hence, by the congruence (18) it follows that $q=p^{e}$ with $1 \leq e \leq f$. By the congruence (6) of Lemma 1 we have

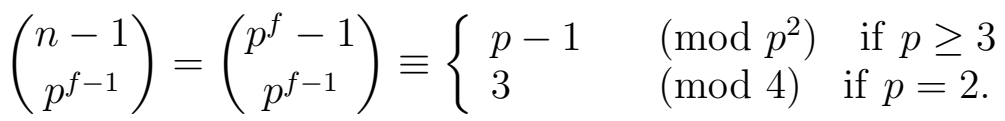

On the other hand, if we suppose that $e \geq 2$, then the congruence (3) with $k=$ $p^{f-1}$ reduced modulo $p^{2}$ yields

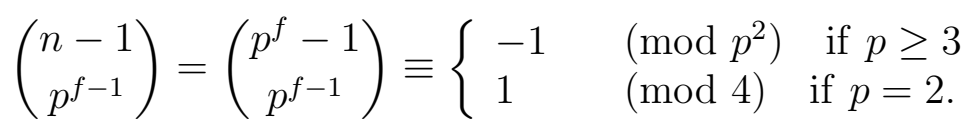

Comparing the congruences $(19)$ and $(20)$, we get $p \equiv 0\left(\bmod p^{2}\right)$. This contradiction shows that must be $e=1$, or equivalently, $q=p$.

Case 3: $s \geq 2$. Then take $s=\sum_{i=0}^{t} s_{i} p^{i}$ with $0 \leq s_{i} \leq p-1$ for all $i=1, \ldots, t$ and $1 \leq s_{0} \leq p-1$. Applying Lucas' theorem (the congruence (4D)), we have

$$
\begin{aligned}
\left(\begin{array}{c}
n-1 \\
p^{f}
\end{array}\right) & =\left(\begin{array}{c}
s p^{f}-1 \\
p^{f}
\end{array}\right)=\left(\begin{array}{c}
\sum_{i=1}^{t} s_{i} p^{i+f}+\left(s_{0}-1\right) p^{f}+\sum_{i=0}^{f-1}(p-1) p^{i} \\
p^{f}
\end{array}\right) \\
& \equiv\left(\begin{array}{c}
s_{0}-1 \\
1
\end{array}\right)=s_{0}-1 \equiv s-1 \quad(\bmod p) .
\end{aligned}
$$

The congruence (21) and the condition (3) with $k=p^{f}$ imply that $s-1 \equiv$ $(-1)^{p^{f}}(\bmod p)$. This shows that must be $s \equiv 0(\bmod p)$. A contradiction, and thus this case is impossible.

Finally, the all three considered cases clearly completes the proof of the theorem. 


\section{REFERENCES}

[1] T.-X. Cai and A. Granville, On the residues of binomial coefficients and their products modulo prime powers, Acta Math. Sinica 18, no. 2, 277-288.

[2] E. Deutsch and I.M. Gessel, Problem 1494, Math. Mag. 70 (April 1997), 143-144.

[3] L. E. Dickson, The History of the Theory of Numbers, vol. 1, Chelsea Publishing, New York, 1966.

[4] C.F. Gauss, Disquisitiones Arithmeticae, Fleischer, Leipzig, 1801.

[5] H.W. Gould and W.E. Greig, A Lucas triangle primality criterion dual to that of MannShanks, Fibonacci Quart. 23, no. 1 (1985), 66-69.

[6] A. Granville, Arithmetic properties of binomial coefficients. I. Binomial coefficients modulo prime powers, in Organic Mathematics-Burnaby, BC 1995, CMS Conf. Proc., vol. 20, American Mathematical Society, Providence, RI, 1997, 253-276.

[7] É. Lucas, Théorie des fonctions numérique simplement périodiques, Amer. J. Math. 1 (1878), 184-240.

[8] H.B. Mann and D. Shanks, A necessary and sufficient condition for primality, J. Combin. Theory Ser. A 13 (1972), 131-134.

[9] R. Meštrović, A Note on the Congruence $\left(\begin{array}{c}n d \\ m d\end{array}\right) \equiv\left(\begin{array}{c}n \\ m\end{array}\right)(\bmod q)$, Amer. Math. Monthly 116 (2009), 75-77.

[10] R. Meštrović, Wolstenholme's theorem: its generalizations and extensions in the last hundred and fifty years (1862-2012); preprint arXiv:1111.3057v2 [math.NT], 2011.

[11] R. Meštrović, An extension of Babbage's criterion for primality, Math. Slovaca 63, no. 6 (2013), 1179-1182.

[12] P.A. Piza, Fermat coefficients, Math. Mag. 27 (1954), 141-146.

Maritime FACUlty, University of Montenegro, Dobrota 36, 85330 Kotor, MonTENEGRO

E-mail address: romeo@ac.me 